BULL. AUSTRAL. MATH. SOC.

VOL. $6(1972), 407-415$.

\title{
A characterization of the Hall planes of odd order
}

\section{P.B. Kirkpatrick}

The Hall projective planes of odd order are characterized in terms of their translations, collineations which fix all the points of a Baer subplane, and involutory homologies.

\section{Introduction}

Let $I$ be a projective plane, $\tau_{\infty}$ a line of $I$ and $I_{0}$ a Baer subplane of $\Pi$ such that $l_{\infty}$ is a line of $\Pi_{0}$. We call II a generalized Hall plane with respect to $l_{\infty}$, $\Pi_{0}$ if

(1) II is a translation plane with respect to $l_{\infty}$, and

(2) II has a group of collineations which is transitive on the points of $l_{\infty}$ not in $\pi_{0}$, and fixes every point in $\Pi_{0}$.

The object of this paper is to prove:

THEOREM. A projective plane $\Pi$ is a Hall plane of odd order if and only if

(a) II is a finite generalized Hall plane with respect to some line $\tau_{\infty}$ and Baer subplane $\pi_{0}$ containing $\tau_{\infty}$, and

(b) each point of $\underline{\underline{M}}=\left\{M \mid M \in Z_{\infty}\right.$ and $\left.M \in \Pi_{0}\right\}$ is the centre of an involutory homology with axis in $\Pi_{0}$.

The necessity of conditions (a) and (b) was proved by Hughes [6]

Received 14 December 1971. 
in 1959.

All finite planes with involutory homologies have odd order.

We use the terminology of Dembowski [2], except that we denote points by capital letters and the coordinate quadrangle $(0,0),(1,1),(0),(\infty)$ by $O, I, X, Y$ instead of $0, e, u, v$.

\section{Preliminary results and lemmas}

If $O, I, X, Y$ is any coordinate quadrangle of a generalized Hall plane $I$, with $0, I, X, Y \in \Pi_{0}$ and $X, Y \in Z_{\infty}$, then the corresponding ternary ring determines a quasifield $F$ which has a sub-quasifield $F_{0}$ such that whenever $z \in F \backslash F_{0}$ and $w \in F$ then $w=z \alpha+\beta$ for exactly one pair $(\alpha, \beta) \in F_{\mathrm{o}} \times F_{\mathrm{o}} . \quad F$ has a group of automorphisms which is transitive on $F \backslash F_{0}$ and fixes every element of $F_{0}$. Consequently, there exist four maps $f, g, h, k: F_{0} \rightarrow F_{0}$ with

$$
(z \alpha) z=z f(\alpha)+\dot{g}(\alpha) \text { and } \beta z=z h(\beta)+k(\beta)
$$

for all $z \in E \backslash F_{0}, \alpha \in F_{0}, \beta \in F_{0}$.

In addition to these facts, we shall need the classification of the subgroups of PSL $(2, q)$ by Dickson ([3], Second Part, Chapter XII), and the following two results:

RESULT 1 (Kirkpatrick [7]). If $\pi$ is a generalized Hall plane of odd order then $F_{0}$ is a field and $F$ is a right vector space (of dimension two) over $F_{0}$ with respect to the operations induced by the quasifield structure of $F$. .

RESULT 2 (André [1]). If $\underline{\underline{H}}_{1}$ and $\underline{\underline{H}}_{2}$ are non-trivial homologies in a finite projective plane, and if $\mathrm{H}_{1}$ and $\mathrm{H}_{2}$ have the same axis but distinct centres, then the group $\left\langle\mathrm{H}_{1}, \underline{\underline{H}}_{2}\right\rangle$ contains a non-trivial elation.

Let us assume throughout the remainder of the paper that $I$ is a generalized Hall plane of odd order, with special line $l_{\infty}$ and special subplane $\Pi_{0}$; and that all coordinate systems mentioned shall have 
$0, I \in \Pi_{0}$ and $X, Y \in \underline{\underline{M}}=\left\{M \mid M \in Z_{\infty}\right.$ and $\left.M \in \Pi_{0}\right\}$. Then $\underline{\underline{M}}=\left\{(\alpha) \mid \alpha \in E_{0}\right\} \cup\{(\infty)\}$ and, by Result 1 ,

$$
(z \alpha+\beta) z=z[f(\alpha)+h(\beta)]+g(\alpha)+k(\beta), \forall z \in F \backslash F_{0}, \alpha \in F_{0}, \beta \in F_{0},
$$

and the four maps are additive homomorphisms.

LEMMA 1. Every elation of $\Pi$ with centre in $\mathrm{M}$ is a translation.

Proof. Suppose there is an elation with centre $M \in M$ which is not a translation. There is an allowable coordinate system with $Y=M$. The non-trivial $(Y, O Y)$-elations are in one-to-one correspondence with the non-zero $d \in F$ such that

$$
x(d+y)=x d+x y, \forall x, y \in F .
$$

If there exists such an element $d$, then, for some $\beta \in F_{0}, \beta \neq 0$, and $z \in F \backslash F_{0}, z(\beta+z)=z \beta+z z$ and $\beta(\beta+z)=\beta \beta+\beta z$. Now

$$
\begin{aligned}
\beta(\beta+z)=\beta \beta+\beta z & \Rightarrow(\beta+z) h(\beta)+k(\beta)=\beta \beta+z h(\beta)+k(\beta) \\
& \Rightarrow h(\beta)=\beta,
\end{aligned}
$$

and

$$
\begin{aligned}
z(\beta+z)=z \beta+z z & \Rightarrow(\beta+z-\beta)(\beta+z)=z \beta+z f(1)+g(1) \\
& \Rightarrow(\beta+z) f(1)+g(1)+(\beta+z) h(-\beta)+k(-\beta)=z[\beta+f(1)]+g(1) \\
& \Rightarrow f(1)+h(-\beta)=\beta+f(1) \\
& \Rightarrow h(\beta)=-\beta .
\end{aligned}
$$

This contradiction establishes the lemma.

LEMMA 2. II has an involutory ( $X, O Y)$-homology if and only if $f:=k=0$ (the zero map).

Proof. The $(X, O Y)$-homologies are given by $(x, y) \leftrightarrow(x b, y)$ where $b \in N_{m}$,

$$
N_{m}=\{b \mid a(b c)=(a b) c ; \forall a, c \in F\}
$$

Suppose $(x, y) \mapsto(x z, y)$, where $z \in F \backslash F_{0}$, is an involutory homology. Then $(x z) z=x$ if $x \in F$, so that $z z=1 ;$ and $z(z \dot{\alpha})=(z z) \alpha=\alpha$ if $\alpha \in F_{0}$, so that $f=0$ and $g(\alpha)=\alpha^{-1}$ if $\alpha \neq 0$. But $g$ is an 
additive homomorphism and $F_{0}$ is a field of odd order, so we have a contradiction.

Now the multiplicative group of $F_{0}$ is cyclic, with unique involution -1 , and it is easily verified that if $f=k=0$ then $-1 \in N_{m}$. Suppose, on the other hand, that $-1 \in N_{m}, \alpha \in F_{0}$ and $z \in E \backslash F_{0}$. Then

$$
\alpha(-z)=(-\alpha) z=-(\alpha z),
$$

and so $(-z) h(\alpha)+k(\alpha)=-[z h(\alpha)+k(\alpha)]$. Thus $k=0$, and, if $\beta \in F_{0}$,

$$
\begin{aligned}
{[(z \alpha+\beta)(-1)]_{z} } & =-[(z \alpha+\beta) z] \\
& =-z[f(\alpha)+h(\beta)]-g(\alpha),
\end{aligned}
$$

whereas

$$
\begin{aligned}
(z \alpha+\beta)(-z) & =[(-z)(-\alpha)+\beta](-z) \\
& =(-z) f(-\alpha)+g(-\alpha)+(-z) h(\beta),
\end{aligned}
$$

that is, $f=0$ also.

LEMMA 3. Suppose $I$ has an involutory $(X, O Y)$-homology $\mathrm{H}_{0, \infty}$ and an involutory $((\alpha), O(\beta))$-homology $\mathrm{H}_{-\alpha, \beta}$, where $\alpha$ and $\beta$ are (distinct) non-zero elements of $F_{0}$. Then $\mathrm{H}_{\alpha, \beta}$ maps $(z)$ to $(-z)$, for all $z$ in $F \backslash F_{0}$, and

$$
g(\tau)=\alpha \beta \gamma h^{-1}\left(\gamma^{-1} \tau\right) \text { for all } \tau \in F_{0},
$$

where $\gamma=2(\alpha-\beta)^{-1}$.

Proof. $\underline{H}_{\alpha, \beta}$ maps $Y$ to $(\sigma)$, where $\sigma=\frac{1}{2}(\alpha+\beta)$, and therefore $\stackrel{H}{=} \alpha, \beta$ maps any affine point $(x, y)$ of $I$ to $\left(x_{1}, y_{1}\right)$, where

$$
y_{1}-x \beta=\left(x_{1}-x\right) \sigma \text { and } y_{1}-y=\left(x_{1}-x\right) \alpha \text {. }
$$

The line $y=x z$ is mapped to a line $y=x w$. Substituting $y=x z$ and $y_{1}=x_{1} w$ in the above equations, we derive:

$$
x_{1} w-x_{1} \sigma=x \beta-x \sigma \text { and } x_{1} w-x_{1} \alpha=x z-x \alpha \text {. }
$$


These imply:

$$
x_{1}=x+(x \beta-x z)(\alpha-\sigma)^{-1} \text { and } x_{1} w=x_{1} \sigma+x(\beta-\sigma) .
$$

Thus

$$
[x+(x \beta-x z) \gamma] \omega=(x \beta-x z) \gamma \sigma+x \beta \text { for all } x \in F
$$

where $\gamma=(\alpha-\sigma)^{-1}=2(\alpha-\beta)^{-1}$.

There exists an automorphism of $F$ which maps $z$ to $w$, while fixing each element of $F_{0}$. Suppose this maps $w$ to $v$. Then we deduce from (1):

$$
[x+(x \beta-x w) \gamma] v=(x \beta-x w) \gamma \sigma+x \beta \text { for all } x \in F \text {. }
$$

But $\underline{\mathrm{H}}_{\alpha, \beta}$ maps $(w)$ to $(z)$, so (1) also yields:

$$
[x+(x \beta-x w) \gamma] z=(x \beta-x w) \gamma \sigma+x \beta \text { for all } x \in F \text {. }
$$

It follows that $v=z$, whence $w=-z+\lambda$ for some $\lambda$ in $F_{0}$.

Now let $G$ be the cyclic group $\left\langle\mathrm{H}_{=0, \infty} \mathrm{H}_{\alpha, \beta}\right\rangle$. There is a homomorphism $\varphi: G \rightarrow\left(F_{0},+\right)$ which maps any $K$ in $G$ to the $\mu$ in $F_{0}$ such that $(z+\mu)=(z)^{\underline{K}}$. We shall prove that $\varphi$ is trivial. Write $p$ equals the characteristic of $F_{0}, q=\left|F_{0}\right|$, and suppose that $G$ contains an element $\underline{\underline{K}}$ of order $p$. Since $\underline{\underline{K}}$ fixes 0 , and $\left(q^{2}-1, p\right)=1, \underline{\underline{K}}$ fixes another affine point $A$ of $\Pi_{0}$. It follows that $K$ is a central collineation with axis $O A$, when restricted to the subplane $\Pi_{0}$. We readily conclude from this that either the two homologies have the same centre or they have the same axis. This contradiction shows that $\left(|G|,\left|F_{0}\right|\right)=1$.

Since $\varphi$ is trivial and $\underline{H}_{0, \infty}$ maps $(z)$ to $(-z), \underline{H}_{\alpha, \beta}$ also maps (z) to $(-z)$.

Substituting $w=-z$, and restricting $x$ to $E_{0}$, we may simplify equation (1) to:

$$
-z h(x+x \beta \gamma)+g(h(x) \gamma)=x \beta(\gamma \sigma+1)-z h(x) \gamma \sigma .
$$


It follows that $g(h(x) \gamma)=x \beta(\gamma \sigma+1)$ for all $x$ in $F_{0}$. Since $\gamma \sigma+1=\alpha \gamma$, the lemma is proved.

\section{Proof of the theorem}

We now assume that each point of $M$ is the centre of an involutory homology with axis in $\Pi_{0}$. The theorem is to be proved by considering separately the cases $q=\left|F_{0}\right| \equiv 3(\bmod 4)$ and $q \equiv 1(\bmod 4)$. We shall refer to the unordered pairs $\{(\alpha),(\beta)\}$ and $\{X, Y\}$ corresponding to the involutory $((\alpha), O(\beta))-,(X, O Y)$ - and $(Y, O X)$-homologies as "special pairs". These special pairs partition $M$ and are permuted by each of the homologies (by Result 2 and Iemma 1).

Case $1: q \equiv 3(\bmod 4)$. The permutation induced by $\underline{\mathrm{H}}_{0, \infty}$ on the set of $\frac{1}{2}(q+1)$ special pairs fixes $\{X, Y\}$ and therefore fixes at least one other special pair $\{(\alpha),(\beta)\}$. The corresponding homologies $\underline{\underline{H}}_{\alpha, \beta}$ and $\mathrm{H}_{\beta, \alpha}$ both interchange $X$ and $Y$. We can change coordinates so that $\stackrel{H}{=}_{\alpha, \beta}$ becomes $\mathrm{H}_{1,-1}$. By Lemma 3 ,

$$
g(\tau)=-h^{-1}(\tau) \text { for all } \tau \in F_{0}
$$

Now consider any $\underline{H}_{\alpha, \beta}$ with $\alpha \neq \pm 1$. Since

${\stackrel{H^{-1}}{=}}_{1,-1}^{-\underline{H}_{\alpha, \beta}} \underline{\underline{H}}_{1,-1}=\underline{\underline{H}}_{\alpha^{-1}, \beta}^{-1}$, we have, from Lerma 3 again

$$
g(\tau)=\alpha \beta \gamma h^{-1}\left(\gamma^{-1} \tau\right)=\alpha^{-1} \beta^{-1}(-\alpha \beta \gamma) h^{-1}\left(-\alpha^{-1} B^{-1} \gamma^{-1} \tau\right),
$$

and so $\alpha \beta h^{-1}\left(\gamma^{-1} \tau\right)=h^{-1}\left(\alpha^{-1} \beta^{-1} \gamma^{-1} \tau\right)$. Putting $\tau=\gamma \rho$, we deduce that

$$
h^{-1}\left(\alpha^{-1}{ }^{-1} \rho\right)=\alpha B h^{-1}(\rho) \text { for all } \rho \in F_{0} \text {. }
$$

It follows that either $\alpha B$ is in the prime subfield GF(p) of $F_{0}$, or $h^{-1}$ induces an involutory automorphism of the extension of $G F(p)$ by $\alpha \beta$. Since $q \equiv 3(\bmod 4)$, the first alternative is the only possibility. Thus $\alpha \beta= \pm 1$, and $g(\gamma)= \pm \gamma h^{-1}(1)= \pm \gamma \quad$ (since $1 . z=z .1$ ), whence $h^{-1}(\gamma)=\mp \gamma$. But $\gamma$ ranges over exactly half of the non-zero elements of 
$F_{0}$, since $\gamma=2(\alpha-\beta)^{-1}$ and if $\gamma^{\prime}=\gamma$ then $\alpha^{\prime} \beta^{\prime}=\alpha \beta$ (Lemma 3). So we may choose a basis for $F_{0}$ (as a vector space over $G F(p)$ ) from among the values taken by $\gamma$, and relative to this basis $h$ has a diagonal matrix whose diagonal entries are \pm 1 .

If one or more of these entries is $-l$, then a contradiction results. For $h(1)=1$ implies that at least one entry is +1 , and so $h$ has only $p^{i}+p^{j}-1$ eigenvectors, for some $i, j$ with $p^{i+j}=p^{n}=q, i>0$, $j>0$. But $h$ has at least $\frac{1}{2}\left(p^{n}-1\right)$ eigenvectors, so $p^{i}+p^{j}-1 \geq \frac{1}{2}\left(p^{n}-1\right)$. Simple calculations now show that (since $p$ is an odd prime) $q=9$, contradicting $q \equiv 3(\bmod 4)$.

Thus $h(\tau)=\tau$ and $g(\tau)=-\tau$ for all $\tau$ in $F_{0}$, that is $F$ is a Hall system.

Case 2: $q \equiv 1(\bmod 4)$. We show first that $\stackrel{H}{0}, \infty_{\infty}$ does not interchange the two points of any special pair. Suppose the contrary. Then choose coordinates so that $\{(1),(-1)\}$ is a special pair. Let $\varepsilon$ be a square root of -1 in $F_{0}$, and $\{(\varepsilon),(\beta)\}$ the corresponding special pair. Each of $\stackrel{H}{=}, \infty_{0}$ and $\stackrel{H}{H}_{1,-1}$ maps this special pair to a special pair. So $\{(-\varepsilon),(-\beta)\}$ and $\left\{(-\varepsilon),\left(\beta^{-1}\right)\right\}$ are special pairs, that is $\beta^{2}=-1$, and $\{(\varepsilon),(-\varepsilon)\}$ is a special pair. By Lemma 3 ,

$$
-h^{-1}(\tau)=g(\tau)=-\varepsilon h^{-1}(\varepsilon \tau) \text { for all } \tau \in E_{0} \text {. }
$$

Thus $h^{-1}(\varepsilon)=-\varepsilon$, and so $\varepsilon$ does not lie in $G F(p)$, and $h^{-1}$ induces an involutory automorphism of the extension of GF $(p)$ by $\varepsilon$. This field contains an element $\rho$ such that $h(\rho) \rho=-1$. But

$$
(z+\rho) z=g(1)+z h(\rho)=-1-z \rho^{-1}=(z+\rho)\left(-\rho^{-1}\right)
$$

if $z \in F \backslash F_{0}$. This contradiction proves our original assertion.

It follows that the collineation group $K$ generated by our involutory homologies is transitive on the set of $\frac{1}{2}(q+1)$ special pairs. 
Let $K^{*}$ be the group induced on the points of $\underline{\underline{M}}$ by $K$, and let $H^{*}$ be the subgroup of $K^{*}$ generated by all the products of two non-trivial elements of $K^{*}$. Then $K^{*}$ is a subgroup of $\operatorname{PSL}(2, q)$, since $q \equiv 1(\bmod 4)$. Also $\left[K^{*}: H^{*}\right]=2$ and $\frac{1}{2}(q+1)|| K^{*} \mid$. But $\frac{1}{2}(q+1)$ is odd, so $q+1|| K^{*} \mid$ and $H^{*}$ is transitive on the set of special pairs.

Now $\left(\left|K^{*}\right|, q\right)=1$, by an argument used in the proof of Lemma 3; also $K^{*} \leq \operatorname{PSL}(2, q)$, and $q+1|| K^{*} \mid$. So either $K^{*}$ is dihedral of order $q+1$ or $K^{*}$ is isomorphic to $A_{4}, S_{4}$ or $A_{5}$ (Dickson [3]). Since $q \equiv 1(\bmod 4), K^{*}$ is dihedral of order $q+1$, and $H^{*}$ is cyclic.

The situation, then, is that our involutory homologies, restricted to the affine portion of $\Pi_{0}$, are the reflections in an orthogonal group $O(2, q)$, and therefore $\alpha \beta$ has the same value for all $\stackrel{H}{=} \alpha, \beta_{\text {. Since }}$ $g(\gamma)=\alpha \beta \gamma$ for all $\gamma$ corresponding to homologies $\underline{H}_{\alpha, \beta}$, an argument used in Case 1 shows that $g(\tau)=\alpha \beta \tau$ for all $\tau$ in $F_{0}$. But $g(\tau)=\alpha \beta \gamma h^{-1}\left(\gamma^{-1} \tau\right)$, and so $h(\tau)=\tau$ for all $\tau$ in $F_{0}$. Thus $F$ is once again a Hall system, and the theorem is proved.

\section{References}

[1] Johannes André, "Über Perspektivitäten in endlichen projektiven Ebenen", Arch. Math. 6 (1954), 29-32.

[2] P. Dembowski, Finite geometries (Ergebnisse der Mathematik und ihrer Grenzgebiete, Band 44. Springer-Verlag, Berlin, Heidelberg, New York, 1968).

[3] Leonard Eugene Dickson, Linear groups, with an exposition of the Galois field theory (B.G. Teubner, Leipzig, 1901; reprinted Dover, New York, 1958).

[4] Marshall Hall, "Projective planes", Trans. Amer. Math. Soc. 54 (1943), 229-277. 
[5] Marshall Hall, Jr, The theory of groups (The Macmillan Company, New York, 1959).

[6] D.R. Hughes, "Collineation groups of non-desarguesian planes, I. The Hall Veblen-Wedderburn systems", Amer. J. Math. 81 (1959), 921-938.

[7] P.B. Kirkpatrick, "Generalization of Hall planes of odd order", Bulz. Austral. Math. Soc. 4 (1971), 205-209.

Department of Pure Mathematics, University of Sydney,

Sydney,

New South Wales. 